\title{
SUR UNE MÉTHODE PUREMENT PHYSIQUE POUR LA DÉTERMINATION DES POIDS MOLÉGULAIRES DES GAZ ET DES POIDS ATOMIQUES DE LEURS ÉLÉMENTS;
}

Par ${ }^{M}$. Daniel BERThelot.

Les équivalents chimiques représentent les proportions pondérales selon lesquelles les corps se combinent.

La méthode la plus directe pour les déterminer est donc la méthode chimique, qui consiste à faire l'analyse ou la synthèse d'un corps composé. On obtient ainsi une première échelle d'équivalents et par suite de poids moléculaires.

Mais on peut employer également une méthode physique, fondée sur la loi de Gay-Lussac, d'après laquelle les volumes des corps qui se combinent, mesurés à l'état gazeux, sont entre eux dans des rapports simples, et sur le principe d'Avogadro-Ampère, qui est une généralisation de cette loi et d'après lequel les volumes moléculaires de tous les gaz sont égaux. Les densités des gaz mesurés à la mème température et sous la même pression fournissent donc une nouvelle échelle de poids moléculaires.

Cependant, en fait, ces deux échelles ne concordent pas exactement; les densités théoriques des gaz, calculées d'après les valeurs des équivalents fournis par l'analyse chimique, ne s'accordent qu'imparfaitement avec les densités expérimentales trouvées dans les conditions ordinaires de température et de pression.

Ce désaccord tient à ce que le principe d'Avogadro-Ampère ne pourrait être exact pour diverses températures et diverses pressions que si tous les gaz avaient même compressibilité et même coefficient de dilatation. Or aucune de ces deux lois n'est rigoureuse.

Mais il résulte des expériences de Regnault que les coefficients de dilatation des divers gaz, tant sous pression constante que sous volume constant, se rapprochent de plus en plus d'une même valeur égale environ à $\frac{1}{273}$. à mesure quela pression diminue. Par suite, les lois de Gay-Lussac sont des lois limites, vraies pour des pressions très faibles. Nous sommes donc autorisés à admettre que, dans ces conditions, de raréfaction extrême, les volumes moléculaires de tous les gaz sont rigoureusement égaux.

L'échelle physique des poids moléculaires, fondée sur les densités 
des gaz, se rapproche donc d'autant plus de l'échelle chimique que la pression est plus faible, et l'on est amené à penser qu'elle se confondrait avec elle si on substituait aux densités normales (c'est-à-dire mesurées à $0^{\circ}$ et sous la pression de l'atmosphère) les densités limites prises sous une pression infiniment faible.

La densité limite d'un gaz ne peut pas être mesurée expérimentalement; mais il est facile de la déduire de sa densité normale, si l'on connaît la loi de compressibilité de ce gaz.

Désignons par $v_{1}$ le volume d'une masse de gaz sous la pression normale $p_{1}$, par $v_{0}$ son volume sous la pression $p_{0}$; si le gaz ne suit pas la loi de Mariotte, nous pouvons poser :

$$
1-\frac{p_{1} v_{1}}{p_{0} v_{0}}=A_{p_{0}}^{p_{1}}\left(p_{1}-p_{0}\right)
$$

$\mathrm{A}_{p_{0}}^{p_{\mathrm{T}}}$ désignant le coefficient moyen d'écart de la compressibilité du gaz par rapport à la loi de Mariotte entre $\mathrm{P}_{0}$ et $\mathrm{P}_{1}$.

Considérons deux gaz $\mathrm{G}_{\mathrm{t}}$ et $\mathrm{G}^{\prime}$ sous une pression infiniment faible $p_{1}$; leurs volumes moléculaires ont une mème valeur $v_{0}$; comprimons ces deux gaz jusqu'à la pression atmosphérique $p_{1}$; leurs volumes moléculaires cessent d'être égaux et prennent les valeurs :

$$
\begin{aligned}
& v_{1}=v_{0} \frac{p_{0}}{p_{1}}\left[1-A_{p_{0}}^{p_{1}}\left(p_{1}-p_{0}\right)\right]=u_{1}\left[1-A_{p_{0}}^{p_{1}}\left(p_{1}-p_{0}\right)\right] \\
& v_{1}^{\prime}=v_{0} \frac{p_{0}}{p_{1}}\left[1-A_{p_{0}}^{p_{1}}\left(p_{1}-p_{0}\right)\right]=u_{1}\left[1-A_{p_{0}}^{p_{1}}\left(p_{1}-p_{0}\right)\right] .
\end{aligned}
$$

$u_{1}$ étant le volume moléculaire que prendraient tous les gaz sous la pression atmosphérique s'ils suivaient la loi de Mariotte.

Si $p_{0}$ est infiniment faible, le rapport de ces volumes moléculaires se réduit à :

$$
\frac{v_{1}}{v_{1}^{\prime}}=\frac{1-A_{0}^{p_{1}} p_{4}}{1-\Lambda_{0}^{p_{1}} p_{1}}
$$

Les volumes moléculaires des divers gaz $\mathrm{G}, \mathrm{G}^{\prime}, \mathrm{G}^{\prime \prime}$ prennent donc, sous la pression $p_{1}$, des valeurs proportionnelles à $1-\mathrm{A} p_{1}$, $1-\mathrm{A}^{\prime} p_{1}, 1-\mathrm{A}^{\prime \prime} p_{1}$. Soient $d_{1}, d_{1}^{\prime}, d_{1}^{\prime \prime}$, leurs densités sous la pression atmosphérique $p_{1}$, leurs poids moléculaires sont égaux aux produits des volumes moléculaires par les densités correspondantes, 
c'est-à-dire proportionnels à $\left(1-\Lambda p_{1}\right) d_{1} \ldots\left(1-\mathrm{A}^{\prime} p_{1}\right) d d_{1}^{\prime} \ldots$ $\left(1-\mathrm{A}^{\prime \prime} p_{1}\right) d_{1}^{\prime \prime}$.

En particulier, si les pressions sont évaluées en atmosphères, $p_{1}=1$, et les densités limites des gaz $\mathrm{G}_{1}, \mathrm{G}_{1}^{\prime}, \ldots$, sont proportionnelles à $\left(1-\mathrm{A}_{0}^{1}\right) d_{1},\left(1-\mathrm{A}_{0}^{\prime 1}\right) d_{1}^{\prime} \ldots$ Tout se réduit donc au calcul du coefficient moyen $A_{0}^{1}$ entre 0 et 1 atmosphère.

Le résultat est immédiat pour les gaz dits autrefois permanents. On sait, par les expériences de Regnault, que, pour ces gaz, le coefficient $\Lambda_{p_{0}}^{p_{\mathrm{I}}}$ reste sensiblement constant pour des variations de pression de 3 à 4 atmosphères. On peut donc sans erreur sensible prendre entre 0 et 1 atmosphère l'écart mesuré entre 1 et 2 atmosphères.

Dans le cas des gaz facilement liquéfiables, le coefficient $A_{p_{0}}^{p_{1}}$ varie assez vite avec $p$, en sorte que, pour calculer $A_{0}^{1}$, il faut tenir compte de la loi de compressibilité du gaz. Or on connaît, d'une manière approchée et plus que suffisante pour le cas actuel, où il ne s'agit que d'une variation de pression relativement faible, la fonction qui lie le volume et la pression d'une masse gazeuse. Non seulement la formule de M. Van der Waals représente bien l'allure générale du phénomène; mais, si l'on détermine ses coefficients de manière à satisfaire exactement aux expériences pour une portion de la courbe de compressibilité, elle donne des valeurs numériques fort bonnes pour les portions voisines.

Nous poserons donc:

$$
p=\frac{\mathrm{RT}}{v-b}-\frac{a}{v^{2}},
$$

$p, v, \mathrm{~T}$ étant la pression, le volume et la température centigrade, augmentée de $273^{\circ}$, de la masse de gaz considérée; $a, b, \mathrm{R}$, trois constantes.

Si l’on choisit pour unité de volume, comme l'a proposé M. Sarrau, le volume qu'occuperait à $0^{\circ}$ et sous la pression normale la masse du gaz, s'il suivait dans ces conditions les lois de Mariotte et de Gay-Lussac (ce qui revient à faire lim. $p v=1$ pour $p=0$ ), $\mathrm{R}$ prend pour tous les gaz la valeur commune 1: 273 et le nombre des constantes caractéristiques de chaque gaz se réduit à deux. Dans le cas actuel, toutes nos données se rapportent à $0^{\circ}$ centigrade, et l'on a $\mathrm{RT}=\mathbf{1}$.

L'équation (2) contenant deux constantes $a$ et $b$, il faut deux conditions pour les déterminer. Comme notre but est de calculer la compressibilité du gaz entre 0 et 1 atmosphère, la première condi- 
tion imposée à la formule sera de représenter exactement la compressibilité du gaz dans la région voisine où elle a été mesurée avec rigueur, c'est-à-dire de donner exactement le coefficient $\mathrm{A}_{2}^{1}$ entre 1 et 2 atmosphères. On fera $p=1, p=2$ dans l'équation ( 2 ), et on calculera les valeurs $v_{1}, v_{2}$, puis l'expression

$$
\frac{v_{1}}{2 v_{2}}-1=\mathrm{A}_{2}^{1}
$$

La seconde condition sera empruntée à l'une des particularités caractéristiques de la courbe de compressibilité; celle qui conduit au calcul le plus simple et qui utilise l'une des constantes des gaz les mieux déterminées consiste à fixer le rapport $\frac{a}{b}$ de manière à retrouver la température critique $t_{c}$ :

$$
\frac{a}{b}=\frac{27}{8} \frac{273+t_{c}}{273}
$$

Le calcul se fait par approximations successives. Sachant que $a$ et $b$ sont toujours petits, on obtient un premier système de valeurs approchées de $a$ et de $b$, en joignant à la valeur $\frac{a}{b}$ donnée par $(3)$, l'expression approchée :

$$
A_{2}^{\prime}=\frac{a--b}{[1-2(a-b)][1-3(a-b)]} .
$$

On obtient d'ailleurs une première valeur approchée de $\mathrm{A}_{0}^{1}$ en posant :

$$
\mathrm{A}_{0}^{\prime}=\frac{a-b}{1-(a-b)}
$$

Si ces valeurs de $A_{0}^{1}$ et $A_{1}^{2}$ étaient rigoureuses, on voit qu'il suffirait de connaître $A_{1}^{2}$ pour en déduire $a-b$ et par suite $A_{0}^{1}$. On n'aurait donc plus besoin de l'équation de Van der Waals, qui aurait seulement servi d'intermédiaire pour le calcul ; quand on fait le calcul complet, on constate que la valeur définitive de $1-\mathbf{A}_{0}^{1}$ diffère de la valeur approchée fournie par l'expression précédente de moins de $\frac{1}{5000}$ pour les gaz moyennement compressibles comme $\mathrm{CO}^{2}$; pour $\mathrm{SO}^{2}$, la différence est 
plus forte et atteint $\frac{1}{2000}$. Ces nombres font voir que, dans la détermination de $A_{0}^{1}$, la valeur de $A_{\uparrow}^{2}$ joue un rôle prépondérant, et que la valeur de $\frac{a}{b}$ donnée par (3) n'exerce que peu d'influence $\left({ }^{1}\right)$.

Il est intéressant néanmoins de remarquer que cette relation (3) paraît assez bien vérifiée par l'expérience, au moins pour les pressions assez faibles (la pression de 1 atmosphère par exemple) dont il est question ici. En effet, des expressions données plus haut il résulte que, pour que les coefficients d'écart A à la loi de Mariotte sous ces faibles pressions s'annulent, il faut que $\frac{a}{b}=1$. L'expression (3) fait donc voir qu'un gaz dont la température critique serait - 192. C. suivrait à $0^{\circ}$ la loi de Mariotte au voisinage de 1 atmosphère. Or M. Leduc, en traçant la courbe empirique des écarts A pour les faibles pressions ( $\frac{1}{76}$ de la pression critique), a trouvé que l'écart serait nul pour un gaz de point critique égal à $-180^{\circ}$. Pour un tel gaz, la densité limite ne différerait pas sensiblement, à $0^{\circ}$ centigrade, de la densité normale.

Les données expérimentales nécessaires au calcul de la densité limite d'un gaz sont $: 1^{\circ}$ sa densité normale; $2^{\circ}$ sa compressibilité au voisinage de la pression atmosphérique; $3^{\circ}$ sa température critique.

Cette dernière donnée n'a que peu de poids sur le résultat final et peut même être laissée entièrement de côté, dans le cas des gaz permanents.

Les densités normales ont été déterminées avec soin par Regnault pour les gaz hydrogène, azote, air, oxygène et anhydride carbonique.

Depuis, lord Rayleigh a signalé une petite correction qu il convient d'apporter aux nombres de Regnault pour tenir compte de la con-

(1) Les isothermes tracés par M. Amagat, en prenant comme coordonnées $p v$ et $p$, permettent une discussion géométrique fort simple du problème et une détermination graphique rapide du résultat. Les valeurs de $A_{0}{ }^{l}$ et $A_{1}{ }^{2}$ ne sont autre chose que les quotients changés de signe des coefficients angulaires moyens des isothermes entre les pressions considérées, par les produits $p_{0} v_{0}$ et $p_{1} v_{1}$. Si l'on possédait un nombre suffisant de valeurs du produit $p v$ au voisinage de la pression atmosphérique, on pourrait peut-être arriver ainsi à une évaluation aussi exacte que par le calcul algébrique. Cette méthode aurait l'avantage de ne rien supposer sur la fonction $f(p, v, \mathrm{~T})$ en dehors de ce que donne l'expérience. 
traction que subit le ballon vide sous l'influence de la pression atmosphérique.

Plus récemment les densités de ces gaz et de divers autres ont été mesurées avec beaucoup de rigueur, d'une manière indépendante, par lord Rayleigh, en Angleterre, et par M. Leduc, en France. Voici les densités trouvées par ces divers expérimentateurs, à $0^{\circ}$ et sous la pression atmosphérique normale :

\begin{tabular}{|c|c|c|c|}
\hline & Regnault (corrigé) & Rayleigh & Leduc \\
\hline Air & 1,00000 & 1,00000 & 1,00000 \\
\hline H & 0,06949 & 川 & 0,06948 \\
\hline 0 & 1,10562 & 1,10335 & 1,10523 \\
\hline $\mathrm{Az}$ & ") & 0,96737 & $0,96 \pi 1 \pi$ \\
\hline $\mathrm{CO}$ & $»$ & 0,96716 & 0,96702 \\
\hline $\mathrm{CO}^{2}$ & 1,52897 & 1,52909 & 1,ว2288 \\
\hline $\mathrm{Az}^{2} \mathrm{O}$ & " & 1,ว2951 & 1,3301 \\
\hline $\mathrm{HCl}$ & $"$ & ” & 1,2692 \\
\hline $\mathrm{C}^{2} \mathrm{H}^{2}$ & $"$ & $»$ & $0,90 \sqsupset 6$ \\
\hline $\mathrm{SO}^{2}$ & ” & ") ( - - - & 2,2639 \\
\hline
\end{tabular}

L'accord de ces divers nombres peut être regardé comme satisfaisant. On remarque pourtant que les nombres de lord Rayleigh sont légèrement supérieur's à ceux de M. Leduc; selon ce dernier savant, cet écart systématique tient à ce que l'air de Londres contiendrait un peu moins d'oxygène que celui de Paris. Il remarque que, si l'on rapporte les densités non pas à l'air, mais à un gaz défini tel que l'oxygène, l'accord a lieu, en général, à $1: 20000$ près; comme j'adopterai plus loin, à l'exemple de la plupart des chimistes, pour base de l'échelle des poids moléculaires, le poids moléculaire de l'oxygène, posé, par convention, égal à 32, ces densités par rapport à l'oxygène sont les seules qui interviendront dans les calculs.

Outre les densités du gaz, il est nécessaire de connaitre leur compressibilité au voisinage de la pression atmosphérique. Les expériences classiques de Regnault portent sur les gaz suivants : air, azote atmosphérique, hydrogène, anhydride carbonique. Les expériences de M. Amagat, qui ont mis en lumière les lois générales de la compressibilité des fluides, ne commencent, en général, qu'à des pressions notablement supérieures à la pression atmosphérique; mais tout récemment MM. Leduc et Sacerdote ont déterminé avec beaucoup de précision les coefficients d'écart d'un grand nombre de gaz 
par rapport à la loi de Mariotte entre 1 et 2 atmosphères (1).

Voici les nombres qu'ils ont trouvés $\left({ }^{2}\right)$ à la température de $16^{\circ}$ :

$\begin{array}{ccccc}\mathrm{H} & \mathrm{Az} & \mathrm{CO} & 0 & \\ -0,00061 & +0,00023 & 0,00030 & 0,00061 & \\ \mathrm{CO}^{2} & \mathrm{Az}^{2} \mathrm{O} & \mathrm{HCl} & \mathrm{C}^{2} \mathrm{H}^{2} & \mathrm{SO}^{2} \\ 0,00526 & 0,00590 & 0,00625 & 0,006.35 & 0,01913\end{array}$

Les coefficients d'écart à la loi de Mariotte donnés ici se rapportent à $16^{\circ}$; les densités données plus haut se rapportent à $0^{\circ}$. Il est donc nécessaire de ramener ces données à une même température. On peut y arriver soit en ramenant les densités à $16^{\circ}$ au moyen des coefficients de dilatation des divers gaz sous la pression atmosphérique, soit en ramenant les coefficients d'écart à $0^{\circ}$ au moyen des coefficients de température appropriés.

M. Leduc a calculé ces derniers coefficients ainsi que les coefficients de dilatation, en s'appuyant sur le principe des états correspondants ; le calcul peut s'appliquer à tous les gaz, sauf à l'hydrogène. Celui-ci se trouvant à l'extrémité de la série, l'expérience directe est indispensable pour savoir comment varie $\mathrm{A}_{o}^{p}$ avec la température. M. Amagat a montré, en 1873, par des expériences faites entre 1 et 2 atmosphères, que, vers $2 \% 0^{\circ}$, l'écart est toujours négatif, mais plus petit en valeur absolue que vers $0^{\circ}$. Cette constatation est d'ailleurs conforme aux prévisions que l'on peut tirer de l'équation de Van der Waals. Il a reconnu plus tard qu'entre $0^{\circ}$ et $100^{\circ}$ les isothermes de l'hydrogène (en prenant comme ordonnées $p v$ et $p$ ) ont sensiblement la forme de droites parallèles dont le coefficient angulaire 0,00072 entre 100 et 1000 atmosphères diffère à peine du coefficient 0,00070 qu'on déduit des mesures de Regnault faites vers $8^{\circ}$ entre 1 et 20 atmosphères et du coefficient 0,00064 observé à $16^{\circ}$ par MM. Leduc et Sacerdote entre 1 et 2 atmosphères. On en conclut que, si $\mathrm{A}_{0}^{1}$ est égal à $-0,00061$ à $16^{\circ}$, il est égal à $-0,00064$ à $0^{\circ}$.

Le tableau suivant contient pour une série de gaz:

$1^{\circ}$ La densité normale $d$, c'est-à-dire à $0^{\circ}$ et sous la pression atmosphérique, du gaz par rapport à l'oxygène; cette densité peut être regardée comme connue à $\frac{1}{10000}$ de sa valeur;

\footnotetext{
(1) Comptes Rendus, 2 août 1897.

() Comme j’adopte ici une unité de pression soixante-seize fois plus forte que ces auteurs (l'atmosphère au lieu du centimètre de mercure), les coefficients de leur tableau sont multipliés par 76.
} 
$2^{\circ}$ La valeur à $0^{\circ}$ du coefficient d'écurt à la loi de Mariotte $A_{2}^{\prime}$ entre 1 et 2 atmosphères, qui est connu d'après MM. Leduc et Sacerdote avec une précision de $\pm 0,00004$ pour les gaz permanents et de $\pm 0,00008$ pour les gaz facilement liquéfiables;

$3^{\circ}$ La température critique du gaz empruntée, en général, aux tableaux publiés par M. Mathias dans l'Annuaire du Bureau des Longiludes $\left({ }^{1}\right)$;

$4^{\circ}, \breve{5}^{\circ}$ Les coefficients $a, b$ de la formule de Van der IVaals, calculés comme il a été dit plus haut; en introduisant ces valeurs de $a$ et de $b$, ainsi que la valeur $\mathrm{R}=\frac{1}{273}$, dans l'équation de Van der Waals appliquée à une masse de chaque gaz égale à sa masse moléculaire, on obtient pour le calcul de la compressibilité des divers gaz au voisinage de la pression atmosphérique des formules préférables à celles qui ont été employées jusqu'ici et dont les constantes étaient calculées au moyen d'expériences moins précises et moins voisines de la pression atmosphérique que celles de MM. Leduc et Sacerdote;

$6^{\circ}$ Le coefficient d'écart de compressibilité $\mathrm{A}_{0}^{1}$ du gaz;

$7^{\circ}$ Le volume moléculaire normal (c'est-à-dire à $0^{\circ}$ et sous la pression atmosphérique) $v_{m}=1-\mathrm{A}_{0}^{1} \mathrm{du}$ gaz ;

$8^{\circ}$ Le poids moléculaire $\mathbf{M}$ du gaz, celui de l'oxygène étant posé, par définition, égal à 32 ,

$$
\frac{\mathrm{M}}{32}=\frac{1-\mathrm{A}_{i}^{0}}{1-\mathbf{A}_{0}^{\prime}} \times \frac{d}{d^{\prime}}
$$

$\mathrm{A}_{0}^{1}$ désignant le coefficient d'écart du gaz, $\mathrm{A}_{0}^{\prime}$ celui de l'oxygène, $d$ la densité normale du gaz, $d^{\prime}$ celle de l'oxygène.

Ces poids moléculaires peuvent être regardés comme déterminés avec une erreur maximum de $\pm \frac{1}{\check{5000}}$.

\begin{tabular}{|c|c|c|c|c|c|c|c|c|c|}
\hline d & $\begin{array}{c}\mathrm{H} \\
0,062865\end{array}$ & $\begin{array}{c}\mathrm{Az} \\
0,87508\end{array}$ & $\begin{array}{c}\mathrm{C} 0 \\
0,87495\end{array}$ & 0 & $\begin{array}{c}\mathrm{CO2} \\
1,38324\end{array}$ & $\begin{array}{r}\mathrm{A} z^{2} 0 \\
1,38450\end{array}$ & $\begin{array}{c}\mathrm{HCl} \\
1,14836\end{array}$ & $\begin{array}{c}\mathrm{C}^{2} \mathrm{H}^{2} \\
0,81938\end{array}$ & $\begin{array}{c}\mathrm{S} 02 \\
2,04835\end{array}$ \\
\hline$A_{1}^{2}$ & $-0,00064$ & $+0,00038$ & 0,00046 & 0,00076 & 0,00692 & 0,00783 & 0,00813 & 0.00866 & 0,02599 \\
\hline$t_{c}$ & $-234^{\circ}(?)$ & $-146^{\circ}$ & $-140^{\circ}$ & $-118^{\circ}$ & $31^{\circ} .35$ & $36^{\bullet}, 0$ & $52^{0}, 0$ & $37^{\circ}, 0$ & $156^{\circ}$ \\
\hline & D & 0.00105 & 0,00118 & 0,00159 & 0,009131 & 0,010244 & 0,010442 & 0,011278 & 0,028400 \\
\hline$\sigma$ & $"$ & 0,00067 & 0,00072 & 0,00083 & 0,002427 & 0,002682 & 0,002599 & 0,002943 & $0,00 \lesssim 350$ \\
\hline$A_{0}^{1}$ & $-0,00064$ & 0,00038 & 0,00046 & 0,00076 & 0,00674 & 0,00761 & 0,00790 & 0,00840 & 0,02358 \\
\hline$v m$ & 1,00064 & 0,99962 & $0,9995 \mathbf{5}$ & 0,99924 & 0,99326 & 0.99239 & 0,99210 & 0,99160 & 0,97642 \\
\hline M & 2,0145 & 28,013 & 28,007 & 32 & 41,000 & 44,000 & 36,486 & 26,020 & 64,050 \\
\hline
\end{tabular}

(1) Pour $\mathrm{CO}^{2}$, j'ai admis le nombre de M. Amagat. 
Conclusions. $-1^{\circ}$ Le volume moléculaire à $0^{\circ}$ et sous la pression atmosphérique étant égal à 1 , pour un gaz qui suivrait exactement la loi de Mariotte, ce volume a la valeur $1-A_{0}^{\prime}$ pour un gaz qui ne la suit pas. Le volume moléculaire de l'hydrogène, qui est moins compressible à $0^{\circ}$ que ne l'exigge la loi, est donc supérieur à 1 ; celui des autres gaz, qui sont plus compressibles, est inférieur à 1 .

Il n'existe qu'un seul cas, où le rapport de deux volumes moléculaires ait été mesuré avec une haute précision : c'est le cas des gaz de l'eau. J'y trouve une confirmation précieuse de mes calculs.

En effet, d'après les valeurs des coefficients $\mathrm{A}_{1}^{2}$, fournis par l'expérience directe à $16^{\circ}$ et donnés plus haut, le rapport des volumes d'hydrogène et d'oxygène, mesurés à $16^{\circ}$ et sous 1 atmosphère, qui se combinent pour former l'eau, est représenté par :

$$
\frac{\mathrm{H}^{2}}{\mathrm{O}}=2,00243 \text {. }
$$

M. Scott ( $\left.{ }^{1}\right)$ a trouvé, par des mesures eudiométriques directes à la température du laboratoire, température qu'il ne précise pas, mais qui paraît avoir été prise voisine de $16^{\circ}$ :

$$
\mathrm{H}^{2}: 0=2,00243 .
$$

$2^{\circ}$ Les poids moléculaires des gaz, simples et composés, donnés plus haut, peuvent servir à calculer leurs poids atomiques. Pour les gaz simples (hydrogène et azote), il suffit évidemment de diviser par 2 les poids moléculaires. On obtient ainsi 1,0072 et 14,007. Pour les gaz composés, oxygénés ou hydrogénés, sachant que $\mathrm{O}=16$ par convention, et que $H=1,0072$, on obtient le tableau suivant:

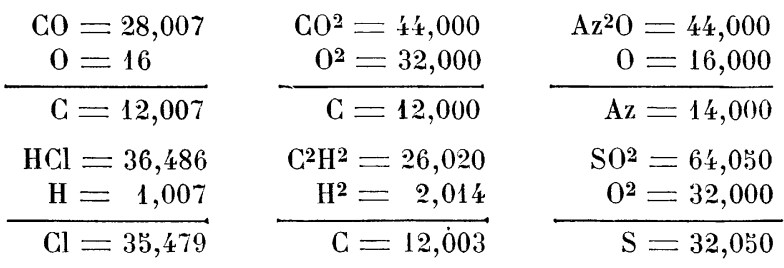

Le poids atomique de l'élément étudié se déduit avec d'autant plus d'exactitude du poids moléculaire du composé correspondant, que cet élément y entre pour une plus forte proportion. Les com-

(1) Proceed. Roy. Soc., t. LIII, p. 130;1893. 
posés hydrogénés sont plus favorables au calcul que les composés oxygénés. En effet, $\mathrm{C}$ forme en poids les $\frac{12}{13}$ de $\mathrm{C}^{2} \mathrm{H}^{2} ; \mathrm{Cl}$, les $\frac{35}{36}$ de $\mathrm{HCl}$; tandis que $\mathrm{S}$ ne forme que $\frac{1}{2}$ de $\mathrm{SO}^{2} ; \mathrm{Az}$, moins de $\frac{1}{3} \operatorname{de~} \mathrm{Az}^{2} \mathrm{O}$, et $\mathrm{C}$ les $\frac{3}{11}$ de $\mathrm{CO}^{2}$. Ce dernier cas est le plus défavorable de tous. L'erreur relative sur le poids atomique du carbone, déduit de la densité de $\mathrm{CO}^{2}$, est presque quadruple de l'erreur qui existe sur cette densité même.

On trouve, en résumé, les poids atomiques suivants :

Hydrogène. - La densité limite de l'hydrogène conduit à $\mathrm{H}=1,00724$, en adoptant pour le rapport des densités de l'hydrogène et de l'oxygène la valeur 0,06286 à de M. Leduc. Si l'on adopte la valeur 0,062892 trouvée par M. Morley, on obtient $\mathrm{H}=1,00772$. La moyenne de ces deux nombres, $\mathrm{H}==1,007 \%$, concorde exactement avec la valeur moyenne qui résulte des nombreuses synthèses de l'eau, faites depuis quelques années.

Carbone. - La densité limite de CO donne 12,007; celle de $\mathrm{CO}^{2}$ donne 12,000 ('); celle de $\mathrm{C}^{2} \mathrm{H}^{2}$ donne 12,003. On peut adopter finalement $\mathrm{C}=12,004$, nombre identique avec celui qui résulte des meilleures synthèses de $\mathrm{CO}^{2}$ par combustion du diamant.

Azote. - La densité limite de Az donne 14,007; la densité limite de $\mathrm{Az}^{2} \mathrm{O}$ donne 14,000. Ce dernier gaz étant plus difficile à obtenir pur et sa densité ayant été l'objet de mesures moins nombreuses que celle de $\mathrm{Az}$, nous attribuons plus de poids au premier nombre, et

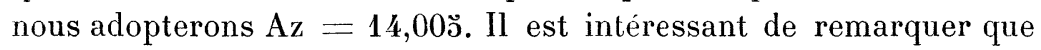
les densités de ces deux gaz nous conduisent, pour le poids atomique de l'azote, à des valeurs concordantes à $\frac{1}{2000}$. Dans le cas de l'azote, en effet, les méthodes chimiques sont fort indirectes et médiocrement concordantes. Stas a obtenu $\mathrm{Az}=14,044$; mais d'autres cycles de réactions donnent des nombres plus bas, tels que 14,019 (Thomsen, 1894) et 14,012 (Hill, 1894).

Argon. - Selon lord Rayleigh $\left(^{2}\right)$ la densité de l'argon par rapport à l'oxygène est 1,24623, et, d'après $M$. Ramsay, ce nombre n'est pas sensiblement modifié par la présence des nouveaux gaz

(1) 12,0033 , si l'on adopte le nombre de lord Rayleigh.

() Proceed. Roy. Soc., t. LXII, p. 209; 1898. 
qu'il a reconnus dans l'air. La compressibilité de l'argon n’a pas été étudiée expérimentalement, mais sachant que ses constantes criticiues sont $t_{c}=-121^{\circ} ; p_{c}=\check{0} 0$ atmosphères, on peut calculer $\mathrm{A}_{0}^{1}$ en appliquant le principe des états correspondants, commel'a fait M. Leduc, c'est-à-dire en traçant la courbe ayant pour abscisses $t_{c}+273$, et pour ordonnées $\mathrm{A}_{0}^{1} p_{\mathrm{s}}$ au moyen des données connues sur les gaz à points critiques voisins $(\mathrm{Az}, \mathrm{CO}, \mathrm{O}, \mathrm{AzO})$ et en relevant sur l'abscisse $t=273^{\circ}-121^{\circ}=192^{\circ}$ la valeur du produit $\mathrm{A}_{0}^{1} p_{c}$ relatif à l'argon . On trouve ainsi $\mathrm{A}_{0}^{1}=0,00070, v_{m}=0,99930, \mathrm{M}=39,882$.

Ch7ore. - La densité limite de $\mathrm{HCl}$ donne $\mathrm{Cl}=\mathbf{3 5 , 4 7 9}$. Stas a trouvé (1) par la synthèse du chlorure et par l'analyze du chlorate d'argent $\mathrm{Cl}=3 \check{\mathrm{g}}, \mathbf{4} 6$.

Ce nombre diffère d'une manière sensible de celui auquel nous arrivons par la méthode des densités limites. Comme ce dernier est déduit de la densité d'un seul composé chloré (HCl), préparé d'une seule manière, il est prudent de faire des réserves pour ce cas.

Soufre. - La densité limite de $\mathrm{SO}^{2}$ conduit à $\mathrm{S}=32,0$ o. Stas a trouré,par la synthèse du sulfure d'argent et par l'analyse du sulfate, $\mathrm{S}=32,008$, nombre qui concorde à moins de $1: 3000$ avec le précédent.

Pour donner une idée de l'erreur que l'on commettrait dans le cas de $\mathrm{SO}^{2}$ en prenant la densité normale au lieu de la densité limite, je dirai que l'on en déduirait $\mathrm{S}=33$, ŏ .

En résumé, les calculs précédents justifient le point de vue dont je suis parli, à savoir que l'hypothèse d'Avogadro et d'Ampère, d'après laquelle: Volumes égaux de tous les gaz renferment même nombre de molécules, est une loi limite qui se vérifie exactement pour de très faibles pressions. Par suite: les poids moléculaires des gaz sont proportionnels à leurs densités limites. Ces densités limiles s'obtiennent en multipliant leurs densilés normales (c'est-a-dire prises $\dot{a} 0^{\circ}$ et sous la pression atmosphérique) par le facteur $1-\mathrm{A}_{0}^{\prime}$, qui représente l'écart de la compressibilité du gas par rapport à celle d'un gaz parfait entre 0 et $\mathbf{1}$ atmosphère.

On obtient ainsi les poids atomiques

$\begin{array}{cccccc}0 & \mathrm{H} & \mathrm{C} & \mathrm{Az} & \mathrm{S} & \mathrm{A} \\ 16 & 1,00 \tau 3 \mathrm{z} & \mathbf{1 2 , 0 0 4} & 14,005 & 32,030 & 39,882\end{array}$

(1) Cf. le calcul systématique des expériences de Stas, donné par M. Van der Plaats dans les Annales de Chimie et de Physique $\left(6^{\circ}\right.$ sćrie, t. VII. 1886) et dans les Comptes Rendas, t. CXVI, 1893.

J. de Pliys., $3^{\circ}$ série, t. VIII. (Mai 1899.) 
Cette méthode, purement physique, pour la détermination des poids atomiques, donne, toutes les fois qu'il est possible de préparer les gaz purs, une précision égale à celle des meilleures méthodes (himiques dans un cas où celles-ci sont directes, et peut l'emporter sur elles dans les cas (comme celui de l'azote) où elles sont indirectes. 